\title{
Evaluation of Nanoporous Gold with Controlled Surface Structures for Laser Desorption Ionization (LDI) Analysis: Surface Area Versus LDI Signal Intensity
}

\author{
Jang Mi Jin, ${ }^{1,3}$ Suhee Choi, ${ }^{2}$ Young Hwan Kim, ${ }^{3}$ Man Ho Choi, ${ }^{4}$ Jongwon Kim, ${ }^{2}$ \\ Sunghwan $\mathrm{Kim}^{1,5}$ \\ ${ }^{1}$ Department of Chemistry, Kyungpook National University, Daegu, Korea \\ ${ }^{2}$ Department of Chemistry, Chungbuk National University, Chungbuk, Korea \\ ${ }^{3}$ Division of Mass Spectrometry Research, Korea Basic Science Institute, Ochang, Korea \\ ${ }^{4}$ Biomolecules Function Research Center, Korean Institute of Science and Technology, Seoul, Korea \\ ${ }^{5}$ Green-Nano-Materials Research Center, Daegu, Korea
}

\begin{abstract}
The structural effect of a nanoporous gold (NPG) surface on the signal intensities of laser desorption ionization-mass spectrometry (LDI-MS) were investigated using NPG surfaces with controlled structures. The relationship between surface area and LDI efficiency was compared and evaluated. Comparisons between bare flat gold and NPG surfaces show that nanostructures increased LDI efficiency. We also found that the LDI signal decreased with increasing depth of nanoporous layers, thus increasing the surface area. This result agrees with a previous report (Shin J. A. et al., J. Am. Soc. Mass Spectrom. 2010, 21, 989) in which the LDI efficiency of small molecules decreased for $\mathrm{ZnO}$ wires with longer lengths. This observation was explained by the penetration and deposition of samples into locations inaccessible to photons because of structural screening. The LDI-MS analysis of oils with NPG surfaces (but without matrix) showed the same trend whereby the NPG with about a $200 \mathrm{~nm}$ depth of porous area showed the highest sensitivity. This study clearly shows that the active surface area for solution chemistry can differ from LDI-MS and that NPGs can function as a substrate for LDI oil analysis.
\end{abstract}

Key words: Mass spectrometry, Nanoporous gold, Laser desorption ionization, Petroleum, Surface area

\section{Introduction}

T aser desorption ionization (LDI) with organic matrix, Ltypically referred to as MALDI is widely used for

Electronic supplementary material The online version of this article (doi:10.1007/s13361-012-0439-2) contains supplementary material, which is available to authorized users.

Correspondence to: Jongwon Kim; e-mail: jongwonkim@chungbuk.ac.kr, Sunghwan Kim; e-mail: sunghwank@knu.ac.kr analysis of biomolecules and polymers. However, this approach is limited for the analysis of a mixture (such as petroleum) since peaks originating from the matrix can be abundant in lower $m / z$ ranges (e.g., $m / z<500$ ) and may overlap with peaks from petroleum. Therefore, developing a method that can be used in conjunction with LDI to improve the ionization efficiency and limit matrix interference would be desirable.

Particles or surfaces with nanostructures have been combined with LDI to improve ionization efficiency and 
reduce matrix interference [1-13]. Although many studies have employed nanostructures to improve LDI efficiency and selectivity, relatively few reports have examined how the nanostructure is related to LDI efficiency [9]. In this study, LDI efficiency obtained from four surfaces with different gold $(\mathrm{Au})$ nanostructures were compared, and the dependence of LDI efficiency on the surface areas was evaluated. Nanoporous Au (NPG) structures adopted in this study as LDI substrates have attracted considerable attention due to their unique properties and possible applications in catalysis [14]. The NPG surface area was controlled by the deposition charge during the electrochemical formation of NPG structures [15]. During the process, discrepancies between surface area and LDI efficiency were investigated. The NPG structures with the best LDI efficiencies were selected and applied for oil analysis.

\section{Experimental}

\section{Preparation of NPG Surfaces}

NPG surfaces were prepared by electrodeposition of $\mathrm{Ag} / \mathrm{Au}$ alloy layers followed by selective dissolution of silver (Ag) in concentrated nitric acid [15]. Detailed information on the preparation of NPGs can be found in previous reports [16]. Briefly, the deposition charge of $0.01,0.02,0.04,0.06$, and $0.08 \mathrm{C}$ was applied to control the thickness of the $\mathrm{Ag} / \mathrm{Au}$ alloy layers at a constant potential of $-0.95 \mathrm{~V}$ (versus $\mathrm{Ag} /$ $\mathrm{AgCl}, 3 \mathrm{MKCl}$ ). For convenience, each substrate prepared with different deposition charge is referred to as NPG \#1 $(0.01 \mathrm{C}), \# 2(0.02 \mathrm{C}), \# 3(0.04 \mathrm{C}), \# 4(0.06 \mathrm{C})$, and \#5 $(0.08 \mathrm{C})$, respectively, for the rest of the manuscript. The alloy layers on $\mathrm{Au} /$ silicon $(\mathrm{Si})$ wafers were transferred to concentrated nitric acid to dissolve Ag. Au nanoplate substrates were electrodeposited on $\mathrm{Au} / \mathrm{Si}$ wafers from a solution containing $15 \mathrm{mM} \mathrm{KAu}(\mathrm{CN})_{2}$ and $0.25 \mathrm{MNa}_{2} \mathrm{CO}_{3}$ at $-1.1 \mathrm{~V}$ with a deposition charge of $0.04 \mathrm{C}$ [16]. Scanning electron microscopy (SEM) characterization was performed using a LEO 1530 Field Emission SEM (Carl Zeiss, Jena, Germany). For cross-sectional SEM images, the backside of the electrodeposited $\mathrm{Au} / \mathrm{Si}$ wafer was scraped with a diamond pencil and split into two parts.

\section{Mass Spectrometry Analysis}

Detailed instrumental parameters for the LDI-time-of-flight (TOF) MS experiment are provided in the Supporting Information. Verapamil hydrochloride (Aldrich, St. Louis, MO, USA) solution of $3 \mu \mathrm{g} / \mu \mathrm{L}$ was prepared in $50 \%$ acetonitrile/water (vol/vol) and diluted 6-fold with $0.1 \%$ TFA in water containing $30 \%$ acetonitrile for LDI analyses. The sample solution $(0.5 \mu \mathrm{L})$ was placed on targets for MS analyses. Oil samples extracted using hydrous pyrolysis and the in situ simulator from rocks collected in the Green River Formation were studied [17]. Difference between the hydrous pyrolysis and the in situ simulator methods was explained in the supporting information. Oil samples were diluted to $1 \mathrm{mg} / \mathrm{mL}$ in $100 \%$ toluene, and $0.5 \mu \mathrm{L}$ of oil solution was spotted on the targets.

\section{Results and Discussion}

\section{Electrochemical Characterization of NPG Surfaces with Different Surface Structures}

Figure 1 shows the SEM images of some of Au target substrates with different surface morphologies used in this study. Figure $1 \mathrm{a}-\mathrm{c}$ show that the NPG substrates with different deposition charges retain regular nanoporous $\mathrm{Au}$ surface structures in which the size of ligaments and pores are around $30 \mathrm{~nm}$ and $20 \mathrm{~nm}$, respectively. The surface area of these NPG substrates were electrochemically estimated by integrating the charges consumed for reduction of the surface oxide monolayer from cyclic voltammograms of NPG substrates obtained from $0.1 \mathrm{M} \mathrm{H}_{2} \mathrm{SO}_{4}$ [18]. A plot showing
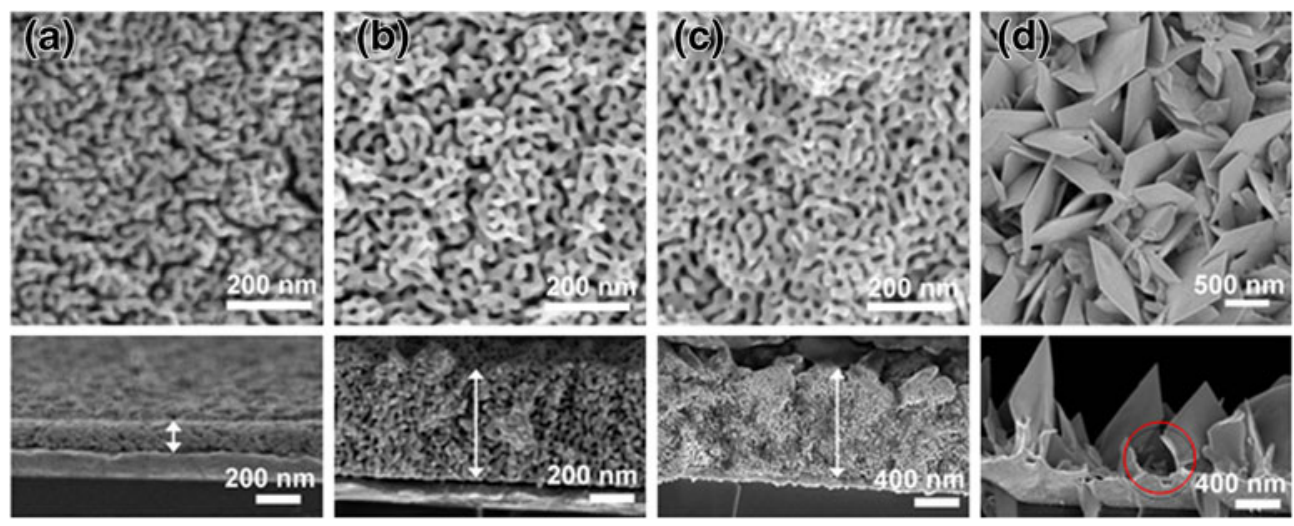

Figure 1. SEM images of Au target substrates with different surface morphologies. The deposition charges for the preparation of $\mathrm{Ag} / \mathrm{Au}$ layers for NPG preparation were (a) $0.01 \mathrm{C}$, (b) $0.04 \mathrm{C}$, and (c) $0.08 \mathrm{C}$. (d) Au nanoplate substrate. Top and bottom rows correspond to the surface and cross-sectional SEM images, respectively. Arrows represent the overall thickness of the electrodeposited nanostructure layers 
Table 1. Average Peak Intensities and Standard Deviations of Verapamil Observed from Five Measurements by Use of NPG Targets

\begin{tabular}{ccc}
\hline NPG target no. & Averaged signal-to-noise ratio & Standard deviation \\
\hline 1 & 590 & 25 \\
2 & 380 & 130 \\
3 & 130 & 46 \\
4 & 94 & 34 \\
5 & 130 & 42 \\
\hline
\end{tabular}

relationship between the relative surface area (RSA) of NPG surfaces normalized with respect to the flat $\mathrm{Au}$ surface and deposition charges is provided in (Figure 1S, Supporting Information). RSA of the NPG substrates with higher deposition charges increased, as reported previously [15]. Figure $1 \mathrm{~d}$ shows the structure of the $\mathrm{Au}$ nanoplate substrates consisting of plate-type protrusions with sharp edges. The RSA of the Au nanoplate substrate was 5.0.

To examine the three-dimensional structures, crosssectional SEM images of the substrates were obtained (bottom of Figure 1). The cross-sectional images show that depths of the porous layer for NPG \#1, \#3, and \#5 increase with higher deposition charges. For example, the depth of the porous layer for NPG \#1 was $<200 \mathrm{~nm}$, but the depth of NPG \#5 was $>800 \mathrm{~nm}$. The structure of the $\mathrm{Au}$ nanoplate consisted of plate-type protrusions with sharp edges, which were confirmed by the cross-sectional image.

\section{Evaluation and Comparison of NPG Surfaces for LDI-TOF-MS}

Equal amounts of verapamil were loaded onto each NPG surface and analyzed by LDI-MS. Examples of LDI-MS spectra of verapamil loaded on different NPG surfaces is presented in Figure 2S (Supporting Information). Ion abundance measured spectra are summarized in Table 1. No signal was observed from the bare Au surface (data not shown) but signals were observed from various NPG surfaces. The fact that no signal was observed from bare $\mathrm{Au}$, but signals could be observed with NPG surfaces, strongly suggests that the nanoporous structure enhances LDI efficiency.

The relative surface areas of the NPG calculated from cyclic voltammograms were 10-60 times higher than that of the flat Au surface. An increased surface area can enhance LDI signals $[1,2,7,8,10]$, but comparisons among NPG surfaces showed differences between surface area and LDI efficiency. Although

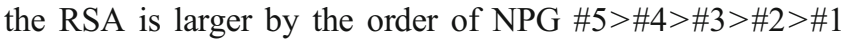
(refer to Figure 1S, Supporting Information), the LDI signal from NPG \#1 was larger than those from NPG \#2 and \#3. No significant difference was observed from NPG \#3, \#4, and \#5. Thus, the increased surface area does not necessarily correlate with LDI efficiency.

Oils samples were loaded on the NPG surfaces without matrix and the obtained LDI-TOF-MS spectra were compared and presented in the supporting information (refer to Figure S3, Supporting Information). The LDI-MS analysis of oils showed the same trend where the NPG \#1 was the

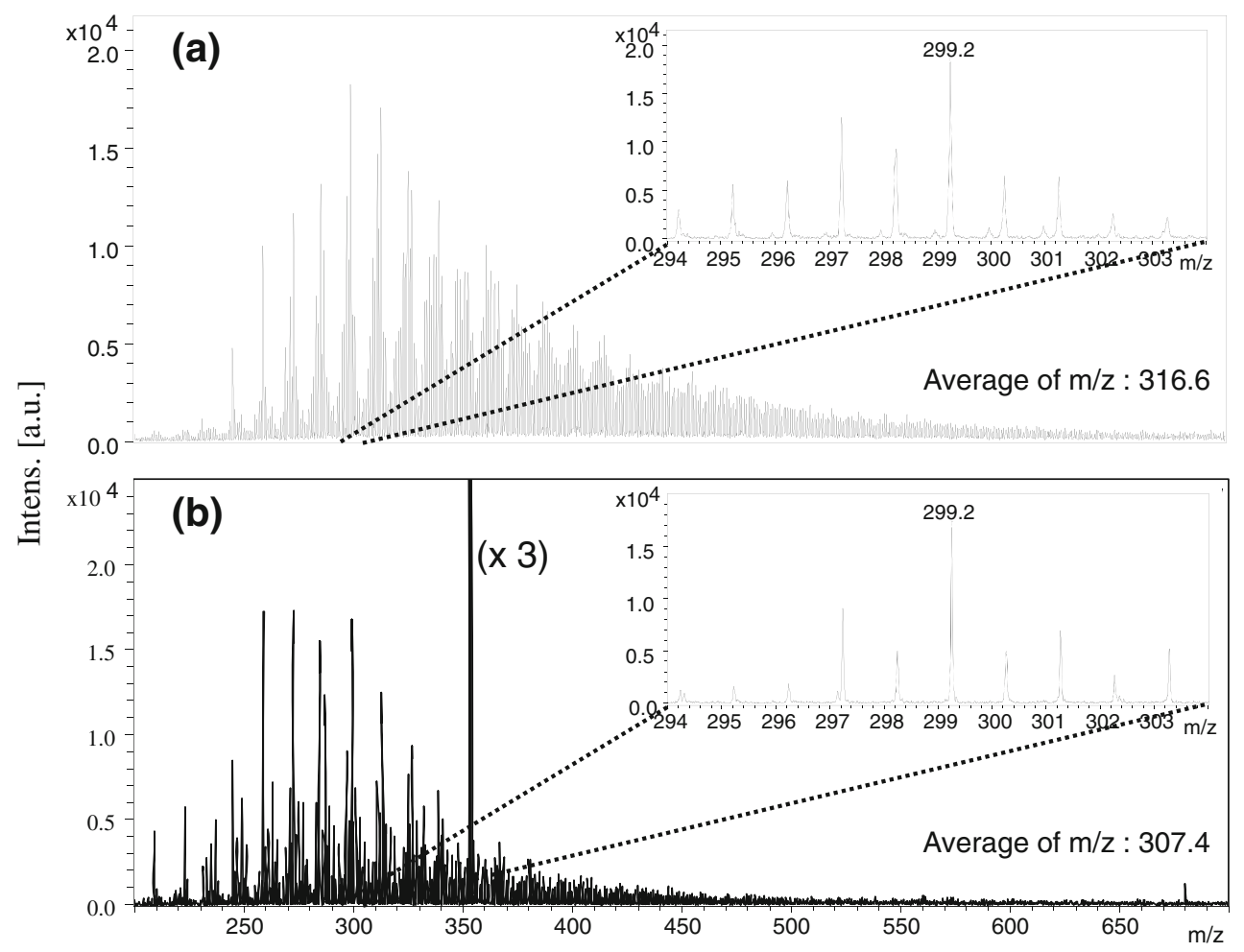

Figure 2. LDI-MS spectra and their expanded view of oil samples each prepared by (a) the hydrous pyrolysis and (b) in situ simulator methods 
most sensitive. NPG \#1 was selected and used for further analyses of oil samples. Oil samples extracted from oil shale rocks by two different methods were loaded onto the NPG plates and analyzed. The obtained spectra are presented in Figure 2. The big peak near $m / z 350$ in Figure $2 \mathrm{~b}$ is originated from crude oil and not from the NPG plates because the peak was found only with the oil shale sample. The average molecular weights can be calculated from the spectra in terms of the magnitude-weighted average as

$$
\text { Molecularweight } t_{\text {average }}=\frac{\sum_{i} I_{i} \times(\text { molecular weight })_{i}}{\sum_{i} I_{i}}
$$

where $\mathrm{I}_{i}$ and (molecular weight) $)_{i}$ are the abundance and molecular weight value of peak $i$, respectively.

The average molecular weights calculated from the spectra obtained from the hydrous pyrolysis and in situ simulator methods are 316.7 and 307.4, respectively. This agrees with a previous study [17] in which a higher molecular weight distribution was observed for samples prepared by hydrous pyrolysis compared to using the in situ simulator method.

The lack of (or even negative) correlation between surface area and LDI efficiency can be partly explained by the deposition of samples onto areas inaccessible by laser photons. Since electrochemical reactions occur in a solution phase, areas partially covered by or located under other structures are accessible to analyte and are electrochemically active. However, this concept does not apply for the LDI experiment. The area partially covered by other structures could be shaded from laser flux and thus inaccessible to photons. For example, if the solution penetrates deeply into the porous layer in Figure $1 \mathrm{~b}$ and $\mathrm{c}$ because of gravity, the laser photons may not be able to reach them. Another example can be found in the SEM image of the $\mathrm{Au}$ nanoplate. The area marked by the red circle in Figure 1 may not be exposed to the laser photon flux because of its structural environment. A previous study reported that signal intensity obtained by LDI gradually decreased as $\mathrm{ZnO}$ nanowire length increased over $250 \mathrm{~nm}$ [9]. They also noted that physical screening using the nanowire could be partly responsible for the observed phenomena. It should be noted that laser shots may change surface morphologies of the substrates. However, since the diameter of laser beam is very small $(\sim 100 \mu \mathrm{m})$, the substrates could be reused up to two or three times without losing reproducibility.

\section{Conclusions}

This study demonstrates that surface morphologies are associated with LDI efficiency. We found that surface area, especially that calculated using solution chemistry, is not correlated with LDI-MS efficiency. Combined with the results of a previous study, photon screening of a nanostructure must be optimized to maximize the efficiency of nanostructure-assisted LDI-MS analysis. Additionally, the nanostructure-assisted LDI-MS analysis may be applicable to oil analysis.

\section{Acknowledgments}

This research was supported by the Basic Science Research Program through the National Research Foundation of Korea (NRF) funded by the Ministry of Education, Science, and Technology (2010-0008441) (S. K.), (2010-0004126) (J. K.), and by the Industrial Strategic technology development program (10038662, MALDI-TOF for the diagnosis of BRCA mutation and genitourinary infection pathogen) funded by the Ministry of Knowledge Economy (MKE, Korea).

\section{References}

1. Wei, J., Buriak, J.M., Siuzdak, G.: Desorption-ionization mass spectrometry on porous silicon. Nature 399, 243-246 (1999)

2. Shen, Z., Thomas, J.J., Averbuj, C., Broo, K.M., Engelhard, M., Crowell, J.E., Finn, M.G., Siuzdak, G.: Porous silicon as a versatile platform for laser desorption/ionization mass spectrometry. Anal. Chem. 73, 612-619 (2001)

3. McLean, J.A., Stumpo, K.A., Russell, D.H.: Size-selected (2-10 Nm) gold nanoparticles for matrix assisted laser desorption ionization of peptides. J. Am. Chem. Soc. 127, 5304-5305 (2005)

4. Castellana, E.T., Russell, D.H.: Tailoring nanoparticle surface chemistry to enhance laser desorption ionization of peptides and proteins. Nano Lett. 7, 3023-3025 (2007)

5. Taira, S., Sugiura, Y., Moritake, S., Shimma, S., Ichiyanagi, Y., Setou, M.: Nanoparticle-assisted laser desorption/ionization based mass imaging with cellular resolution. Anal. Chem. 80, 4761-4766 (2008)

6. Woo, H.-K., Northen, T.R., Yanes, O., Siuzdak, G.: Nanostructure-initiator mass spectrometry: A protocol for preparing and applying NIMS surfaces for high-sensitivity mass analysis. Nat. Protoc. 3, 1341-1349 (2008)

7. Chen, W.-T., Chiang, C.-K., Lin, Y.-W., Chang, H.-T.: Quantification of captopril in urine through surface-assisted laser desorption/ionization mass spectrometry using 4-mercaptobenzoic acid-capped gold nanoparticles as an internal standard. J. Am. Soc. Mass. Spectrom. 21, 864-867 (2010)

8. Gulbakan, B., Park, D., Kang, M., Kececi, K., Martin, C.R., Powell, D.H., Tan, W.: Laser desorption ionization mass spectrometry on slicon nanowell Arrays. Anal. Chem. 82, 7566-7575 (2010)

9. Shin, W.J., Shin, J.H., Song, J.Y., Han, S.Y.: Effects of Zno Nanowire Length on Surface-Assisted Laser Desorption/Ionization of Small Molecules. J. Am. Soc. Mass. Spectrom. 21, 989-992 (2010)

10. Kim, Y.-K., Na, H.-K., Kwack, S.-J., Ryoo, S.-R., Hong, Y.L.S., Hong, S., Jeong, Y., Min, D.-H.: Synergistic effect of graphene oxide/ MWCNT films in laser desorption/ionization mass spectrometry of small molecules and tissue imaging. ACS Nano 5, 4550-4561 (2011)

11. Pavlásková, K.I., Strnadová, M., Strohalm, M., Havlíček, V.r., Šulc, M., Volný, M.: Time-dependent oxidation during nano-assisted laser desorption ionization mass spectrometry: A useful tool for structure determination or a source of possible confusion? Anal. Chem. 83, 5661-5665 (2011)

12. Shrivas, K., Hayasaka, T., Sugiura, Y., Setou, M.: Method for simultaneous imaging of endogenous low molecular weight metabolites in mouse brain using Tio2 nanoparticles in nanoparticle-assisted laser Desorption/Ionization-Imaging Mass Spectrometry. Anal. Chem. 83, 7283-7289 (2011)

13. Yoshioka, K., Ando, D., Watanabe, T.: A comparative study of matrix- and nano-assisted laser desorption/ionization time-of-flight mass spectrometry of isolated and synthetic lignin. Phytochem. Anal. 23, 248-253 (2011)

14. Wittstock, A., Zielasek, V., Biener, J., Friend, C.M., Baumer, M.: Nanoporous gold catalysts for selective gas-phase oxidative coupling of methanol at low temperature. Science 327, 319-322 (2010)

15. Seo, B., Kim, J.: Electrooxidation of glucose at nanoporous gold surfaces: Structure, dependent electrocatalysis, and its application to amperometric detection. Electroanalysis 22, 939-945 (2010)

16. Seo, B., Choi, S., Kim, J.: Simple electrochemical deposition of Au nanoplates from $\mathrm{Au}(\mathrm{I})$ cyanide complexes and their electrocatalytic activities. ACS Appl. Mater. Interfaces 3, 441-446 (2011)

17. Jin, J. M., Kim, S., Birdwell, J. E.: Molecular characterization and comparison of shale oils generated by different pyrolysis methods. Energy Fuels 26, 1054-1062 (2012). doi:10.1021/ef201517a

18. Trasatti, S., Petrii, O.A.: Real surface-area measurements in electrochemistry. Pure Appl. Chem. 63, 711-734 (1991) 